\title{
Right idiopathic popliteal aneurysm in a 5-year-old boy: Case report
}

\author{
Axel Tolstano ${ }^{1,2}$, Edward Lozano-H. ${ }^{1,2}$, Silvio Cohen-Rios ${ }^{1}$, Geanina Lora-Thomas ${ }^{1}$, \\ Hanner Acevedo-Reyes ${ }^{1}$, Laura Aguilera ${ }^{1}$, and Ronald Maestre-Serrano ${ }^{1 *}$ \\ ${ }^{1}$ Faculty of Health Sciences, Universidad Libre Seccional Barranquilla; ${ }^{2}$ Department of Vascular Surgery, Organización Clínica General del Norte. \\ Barranquilla, Colombia
}

\begin{abstract}
We describe the case of a 5-year-old boy with an idiopathic popliteal artery aneurysm on the right leg, who presented the first clinical manifestations from the age of 3 months, with an increase in the circumference of the right lower limb, visible superficial venous system and purplish coloration, without compromised mobility. A final imaging diagnosis was made when patient was 2 years old, through computed tomography angiography, with subsequent surgical repair at 5 years by popliteal-popliteal bypass (supra-infra patellar), bypass bridge with reversed greater saphenous vein, anastomosis with continuous stitches, proximal, and distal ligation of the aneurysm. Patient evolves satisfactorily in the post-operative period.
\end{abstract}

Key words: Popliteal artery. Angioedema. Aneurysm.

\section{Introduction}

In adults, popliteal artery aneurysm is the second most common type of peripheral arterial aneurysm after aortoiliac aneurysm. Atherosclerosis is its most common etiology, while less common causes include trauma, congenital popliteal aneurysm, mycotic aneurysm, and inflammatory arteritis ${ }^{1}$. While $70 \%$ of peripheral aneurysms in the adult population are popliteal, the prevalence within the pediatric population remains at only $1 \%^{2}$. Non-traumatic arterial aneurysms in the pediatric population are very rare and usually associated with inflammatory or degenerative conditions ${ }^{3}$. The most frequent location is the thoracic or abdominal aorta, being rare in other locations ${ }^{4}$. Peripheral aneurysms and pseudoaneurysms have been described in patients with inherited connective tissue diseases that weaken the structure of vascular tissue, such as Ehlers-Danlos and Marfan syndrome ${ }^{5}$. Aneurysm formation is also described in cases of trauma or in diseases such as fibromuscular dysplasia, osteochondroma, infection, and vasculitis ${ }^{6-8}$. However, isolated idiopathic popliteal artery aneurysms are exceptionally rare in the pediatric population. If surgical repair is not performed, popliteal artery aneurysms can lead to several severe complications. This is the sixth case reported and the youngest patient.

\section{Case report}

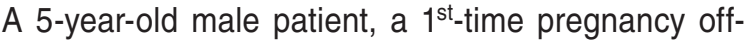
spring, mother was 18 years old at the time. No complications during gestation and born at term by caesarean section due to cephalopelvic disproportion; presenting clinical signs from the age of 3 months, with alterations in the morphology of the right lower limb such as an increase in the diameter of the limb, purplish coloring, and dilation of the venous system, becoming
Correspondence:

*Ronald Y. Maestre-Serrano

E-mail: ronaldy.maestres@unilibre.edu.co

0185-1063/@ 2020 Sociedad Médica del Hospital General de Mexico. Published by Permanyer. This is an open access article under the CC BY-
Date of reception: $27-05-2020$

Date of acceptance: $29-07-2020$

DOI: 10.24875/HGMX.20000060
Available online: 19-04-2021

Rev Med Hosp Gen Mex. 2021:84(2):75-79

www.hospitalgeneral.mx NC-ND license (http://creativecommons.org/licenses/by-nc-nd/4.0/). 
visible. The first study performed was an arterial and venous Doppler ultrasound of the lower limbs; no pathological findings were found, but the result was not compatible with the patient's clinical condition. Other extension studies were considered necessary to guide the diagnosis and explain the anatomical alterations present in the patient's limb.

When the patient was 2 years old, computed tomography angiography (CTA) of the lower limbs was performed, which reported fusiform dilatation of the right popliteal artery in length at $47 \mathrm{~mm}$, anteroposterior and transverse diameter of $12 \times 11 \mathrm{~mm}$ and permeable, aneurysm only affects the popliteal artery. The patient was followed up periodically at an outpatient clinic.

At 5 years of age, patient underwent another CTA, confirming the existence of popliteal aneurysm, without significant variations in the size of the aneurysm described (Fig. 1); the patient was admitted to the institution referred by his attending physician, who reported an increase in the perimeter of the limb involved in the previous year. Physical examination revealed a congestive, purplish-colored right lower limb with increased circumference, distal pulses present, popliteal pulse more evident than the left, with no gait disturbance, or intermittent claudication (Fig. 2).

During the surgery, a longitudinal incision was made in the inner side of the left thigh to extract the supragenicular left greater saphenous vein, which was prepared as a bypass graft. A longitudinal incision was made in the distal third of the right thigh, medial aspect along the anterior edge of the sartorius muscle, on the lower edge of the vastus medialis muscle. Adequate visualization was achieved without releasing muscle bundles, dissection, and repair of the supragenicular right popliteal artery, identifying the start of the aneurysm (Fig. 3). Similarly, a longitudinal incision of $1-2 \mathrm{~cm}$ was performed posterior to the medial edge of the tibia. Dissection and repair of the same artery at the infragenicular level. Tunneling was performed inferior to the muscle fascia to interpose reversed greater saphenous vein between the popliteal segments described (Fig. 4). Proximal and distal veno-popliteal end-lateral anastomosis was performed with continuous Prolene stitches (7:0), thus completing popliteal bypass $^{9}$. Proximal and distal ligation of the popliteal aneurysm was performed. Hemostasis was also performed with teseel $4 \mathrm{ml}$, and a sample of the aneurysm was obtained for pathological study. It was decided not to conduct total resection of the aneurysm as it would result in increased surgical time, risk of injury to surrounding structures, the need to release muscle

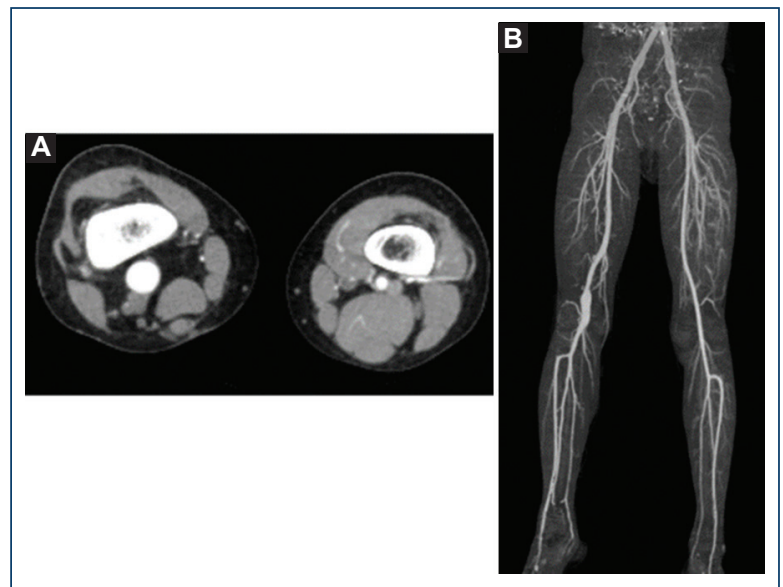

Figure 1. A: computed tomography angiography (CTA) of lower limbs, cross-section showing difference in diameters between the right popliteal artery and the normal left popliteal artery. B: 3D reconstruction of lower limb - CTA.

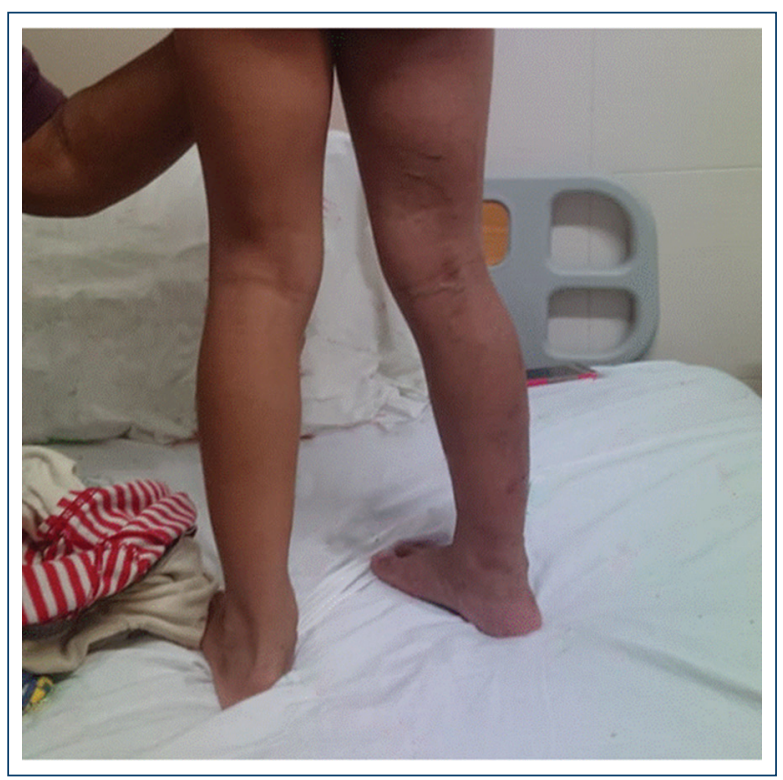

Figure 2. Right lower limb with purplish discoloration, visible superficial venous system, and increased circumference.

bundles and because it would not bring benefits to the patient.

The patient evolved satisfactorily in the immediate postoperative period at the pediatric intensive care unit, where recovery protocols continued and 2 days after surgery patient was transferred to the hospital ward. On physical examination, the pediatric pulses were present, capillary filling and temperature of the 


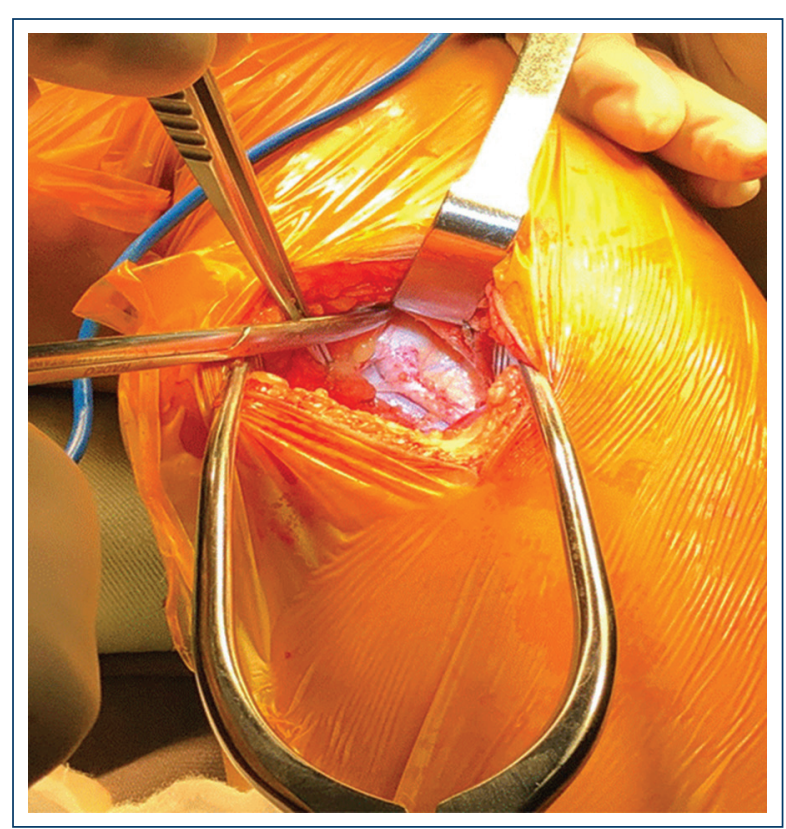

Figure 3. Dissection of supragenicular right popliteal artery, proximal end of aneurysm is evident.

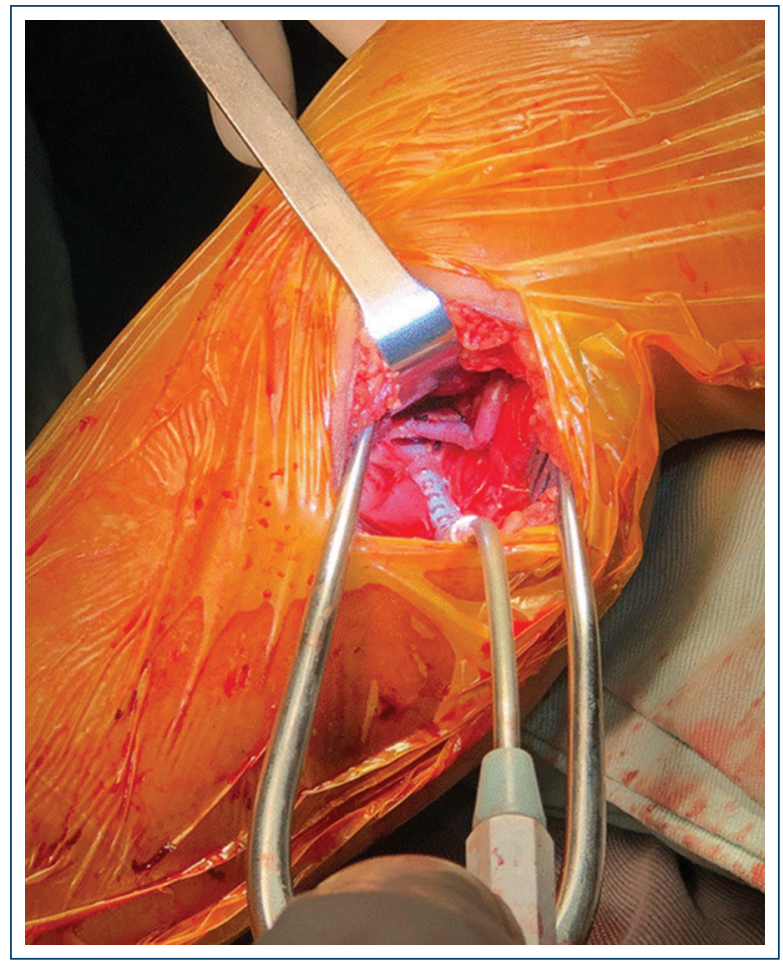

Figure 4. Bypass with redundant reverse great saphenous vein, considering the normal growth of the patient and therefore of the limb, prolonging the life of the bypass.

extremity were preserved, the gait was antalgic and there were no alterations in mobility.
Finally, the pathology report of the biopsy taken from the aneurysm does not describe any pathological findings.

\section{Discussion}

Arterial aneurysm is defined as an abnormal dilation of a blood vessel. A true aneurysm involves symmetrical dilation of all three layers of blood vessels while preserving the histological structure. When a diagnosis of an aneurysm is made, a complete workup of abdominal aneurysms or additional aneurysms of the extremities should be performed. False aneurysm, or pseudoaneurysm, is a solution of continuity of an arterial structure, which is contained. Pseudoaneurysm is caused by a rupture in the arterial wall, which leads to extravasation of blood into the surrounding connective tissue and usually follows injury or iatrogenic trauma. Both true and false aneurysms can occur in any blood vessel in the body, although they are most common in the central and intracranial major vessels ${ }^{10}$.

True aneurysms are commonly caused by atherosclerosis and hypertension; however, this only applies to the adult population ${ }^{10}$. In 1991, the clinicopathological classification of nine categories of arterial aneurysms in pediatric patients was published: (I) arterial infection; (II) giant cell arteritis; (III) autoimmune vasculitis; (IV) Kawasaki disease; (V) medial layer degeneration (Ehlers-Danlos or Marfan syndrome); (VI) other forms of medial layer degeneration; (VII) arterial dysplasia; (VIII) idiopathic congenital factors; and (IX) extravascular events leading to pseudoaneurysm ${ }^{11}$. Following this classification, the aneurysm of the patient under study was classified as type VIII, since he had no family history of connective tissue diseases, autoimmune vasculitis, history of trauma, and rheumatic fever or Kawasaki disease. This includes both signs and symptoms. In addition, no other aneurysmal lesion was evidenced in the extension studies. The pathology report of the biopsy taken from the aneurysm does not describe pathological findings, so it is compatible with this classification.

Aneurysms may manifest as asymptomatic or present as a pulsatile mass with shivering, local pain and ischemic pain ${ }^{10}$. In this case, the patient presented with venous congestion, visible superficial venous system and edema with increased circumference of the right lower limb with respect to the contralateral limb, clinical signs that had not been reported in the cases previously described so far in the literature $3,4,8,12,13$. 
Diagnosis is made by clinical examination and complementary tests such as echo-Doppler, angiography, computed axial tomography or magnetic resonance angiography. Possible complications include rupture of the aneurysm, distal thrombosis, which can lead to loss or delayed growth of the limb, indicating that it should be managed surgically ${ }^{10}$.

Cases reported in the literature on true idiopathic popliteal aneurysms in children are very rare $\mathrm{r}^{3,4,8,12,13}$. To the best of our knowledge, there are only five cases worldwide, two of which occurred in adolescent patients $^{12,13}$. Of the five cases, one was repaired with a vascular prosthesis, which was motivated by aesthetic reasons, taking into account that the use of the greater saphenous vein for the shunt required an additional incision, altering the appearance of the contralateral $\operatorname{limb} b^{12}$. However, the other cases have been performed with reversed greater saphenous vein, bearing in mind the technical considerations described in the literature, which emphasize the disadvantages of vascular prostheses, highlighting the deficiency in patency rates due to the lack of endothelium. This rate decreases as the length of the vascular prosthesis increases, which is counterproductive in pediatric cases, as it is recommended to perform the shunt with an additional length to favor adaptation to the patient's growth, in addition to the risk of lifelong infection that is considered with these grafts ${ }^{11}$. Conversely, there are case reports describing durability of up to 44 years in bypasses, using greater saphenous vein in pediatric patients ${ }^{14}$; therefore, the use of greater saphenous vein grafts is argued based on their correct adaptation of the bypass to the growth of the limb and longer useful life than the synthetic vascular prosthesis.

In this case, the greater saphenous vein of the contralateral limb was taken because of venous congestion in the affected limb, which increases the risk of bleeding.

Endovascular repair of popliteal aneurysm with stenting is one of the most widely accepted surgical alternatives in recent years in the adult population; however, there are no reported cases of this technique in pediatric patients. Children, due to their high physical activity, perform extensive skeletal movements that could constantly produce exaggerated bending and twisting of the stent ${ }^{15}$. Therefore, it would be a risk to use stents in infants, given the imminent danger of stent fracture, increasing the likelihood of reoperation for stent repair.

Only in one of the five previously known cases, separate stitches were used for the anastomosis, following the technical considerations of vascular surgery in the pediatric population mentioned above, where they also suggest absorbable suture to allow better radial growth of the intervened vascular structure ${ }^{11}$. A hypothesis that has not been supported by studies in the literature; in the other cases of idiopathic popliteal artery aneurysm in the pediatric population mentioned, anastomosis was performed with non-absorbable suture and using continuous stitches, obtaining good results $3,4,12,13$.

In the cases reported in the literature, aneurysm resection is performed $d^{3,4,12,13}$. However, in the present case, it was decided not to perform such resection and it was managed with proximal and distal ligation, following the conduct taken in other cases reported in the literature ${ }^{8}$, seeking to obtain a shorter surgical time, less risk of injury to surrounding structures, and avoiding the need to release muscle bundles.

The present clinical case is relevant for several reasons; the first is the low frequency of this condition in the pediatric population, with scarce and limited information in the medical literature for its management, with a clinical presentation not previously reported and from a very early age. In addition, the importance of exhaustive anamnesis, thorough physical examination and an adequate diagnostic imaging study is emphasized, allowing for differentiation of this condition from other vascular pathologies. Finally, the aim was to highlight the surgical variations during management, considering those used in the cases already published; thus, widening the window of possibilities for progress in the treatment of these patients. Furthermore, depending on the clinical evolution, to document the long-term benefits of these modifications, considering the growth of the limb, the behavior of the bypass and the absence of complications, improving the patient's living conditions, allowing us to propose this technique as the most appropriate for the management of this pathology, which is still under study at present.

\section{Conflicts of interest}

The authors declare that there are no conflicts of interest.

\section{Ethical disclosures}

Protection of human and animal subjects. The authors declare that no experiments were performed on humans or animals for this study. 
Confidentiality of data. The authors declare that they have followed the protocols of their work center on the publication of patient data.

Right to privacy and informed consent. The authors declare that no patient data appear in this article.

\section{References}

1. Verikokos C, Karaolanis G, Doulaptsis M, Kouvelos G, Kotzadimitriou A Palla V, et al. Giant popliteal artery aneurysm: case report and review of the literature. Case Rep Vasc Med. 2014;2014:780561.

2. Trickett JP, Scott RA, Tilney HS. Screening and management of asymptomatic popliteal aneurysms. J Med Screen. 2002;9:92-3.

3. Hurley PR, Giddings AE. Idiopathic true aneurysm of the popliteal artery in childhood. Cardiovasc Surg. 1994;2:381-3.

4. Olguín R, Marín J, Seymour C, Werner K, Covarrubias G, Villablanca M et al. Aneurisma poplíteo en niño. Rev Chil Cir. 2016;68:170-2.

5. Davis FM, Eliason JL, Ganesh SK, Blatt NB, Stanley JC, Coleman DM. Pediatric nonaortic arterial aneurysms. J Vasc Surg. 2016;63:466-76.e1.

6. Kayssi A, Shaikh F, Roche-Nagle G, Brandao LR, Williams SA, Rubin BB Management of acute limb ischemia in the pediatric population. $\mathrm{J}$ Vasc Surg. 2014;60:106-10
7. Guvendik L, Bloor K, Charlesworth D. Popliteal aneurysm: sinister harbinger of sudden catastrophe. Br J Surg. 1980;67:294-6.

8. Sivaharan A, Elsaid T, Stansby G. Acute leg ischaemia in a child due to a thrombosed popliteal aneurysm. EJVES Short Rep. 2019;42:1-3.

9. Bensaid MB, Ouldsalek E, Cheysson PE, Kechabtia K, Feito B, Davaine JM. An aneurysmal degeneration of venous bypass for popliteal artery entrapment syndrome. Ann Vasc Surg. 2016;36:296.e1-4.

10. Fredes SC, Franco JR, Fernández VJ, Arambrun MB, Díaz AO, Contreras TJ. Aneurisma verdadero de arteria humeral en lactante. Rev Chil Cir. 2002;54:532-5.

11. Peter SD, Ostlie DJ. A review of vascular surgery in the pediatric population. Pediatr Surg Int. 2007;23:1-10.

12. Jadhav S, Sanagar S, Kaushik S, Munde H. Giant popliteal artery aneurysm in a teenager: an unusual occurrence. Korean J Thorac Cardiovasc Surg. 2019;52:116-8.

13. Notrica DM, Amaro E, Linnaus ME, Zoldos J. Unusual cause of acute lower extremity ischemia in a healthy 15-year-old female: a case report and review of popliteal artery aneurysm management in adolescents. $J$ Pediatr Surg Case Rep. 2016;14:8-11.

14. Pradka SP, Akbari CM, Ricotta JJ, Keshishian JM. Durability of saphenous vein grafts: 44 -year follow-up of a saphenous vein interposition graft in a pediatric patient. J Vasc Surg. 2012;56:216-8.

15. Chang IS, Chee HK, Park SW, Yun IJ, Hwang JJ, Lee SA, et al. The primary patency and fracture rates of self-expandable nitinol stents placed in the popliteal arteries, especially in the P2 and P3 segments, in Korean patients. Korean J Radiol. 2011;12:203-9. 